\title{
A GENERALIZATION OF TORRES' SECOND RELATION
}

\author{
BY
}

\author{
LORENZO TRALDI
}

\begin{abstract}
Let $L=K_{1} \cup \cdots \cup K_{\mu}$ be a tame link in $S^{3}$ of $\mu>2$ components, and let $L_{\mu}$ be its sublink $L_{\mu}=L-K_{\mu}$. Let $H$ and $H_{\mu}$ be the abelianizations of $\pi_{1}\left(S^{3}-L\right)$ and $\pi_{1}\left(S^{3}-L_{\mu}\right)$, respectively, and let $t_{1}, \ldots, t_{\mu}$ (resp., $\left.t_{1}, \ldots, t_{\mu-1}\right)$ be the usual generators of $H$ (resp., $H_{\mu}$ ). If $\phi: \mathbf{Z H} \rightarrow \mathbf{Z} H_{\mu}$ is the (unique) ring homomorphism with $\phi\left(t_{i}\right)=t_{i}$ for $1<i<\mu$, and $\phi\left(t_{\mu}\right)=1$, then Torres' second relation is equivalent to the statement that $\phi E_{1}(L)=\left(\left(\mathbb{I}_{i<\mu} t_{i}^{t^{i}}\right)-1\right) \cdot E_{1}\left(L_{\mu}\right)$, where for $1<i<\mu, l_{i}$ is the linking number $l_{i}=l\left(K_{i}, K_{\mu}\right)$. We prove that if $I H_{\mu}$ is the augmentation ideal of $\mathbf{Z H}_{\mu}$, then for any $k>2$,

$$
E_{k-1}\left(L_{\mu}\right)+\left(\left(\prod_{i<\mu} t_{i}^{4}\right)-1\right) \cdot E_{k}\left(L_{\mu}\right) \subseteq \phi E_{k}(L) \subseteq E_{k-1}\left(L_{\mu}\right)+I H_{\mu} \cdot E_{k}\left(L_{\mu}\right),
$$

and examples are given to indicate that either of these inclusions may be an equality. This theorem is used to generalize certain known properties of $E_{1}$ to the higher ideals.
\end{abstract}

1. Introduction. Let $L=K_{1} \cup \cdots \cup K_{\mu} \subseteq S^{3}$ be a tame link with $\mu>1$ components, and let $G=\pi_{1}\left(S^{3}-L\right)$ be the group of $L, H$ its abelianization. Then $H$ is the (multiplicative) free abelian group generated by certain elements $t_{1}, \ldots, t_{\mu}$ which are defined geometrically (see §2), and its integral group ring $\mathbf{Z H}$ consists of polynomials (with integer coefficients) in the $t_{i}$ and their inverses. In particular, $\mathbf{Z H}$ is a unique factorization domain, and hence a g.c.d. ring.

The elementary ideals $E_{k}(L)$ are defined (in \$2) for every $k \geqslant 1$; they are ideals of the ring $\mathbf{Z} H$, and form an ascending sequence of invariants of $L$. These ideals have g.c.d.s $\Delta_{k}(L)=$ g.c.d. $E_{k}(L)$, which are also invariants of $L$. In general, the polynomials $\Delta_{k}(L)$ are weaker invariants than the ideals $E_{k}(L)$; however, it is well known that $\Delta_{1}(L)$, the Alexander polynomial of $L$, determines $E_{1}(L)$ by the following property:

$\left(1_{1}\right)$ if $\mu=1$ then $E_{1}(L) \subseteq \mathbf{Z H}$ is the principal ideal $E_{1}(L)=\left(\Delta_{1}(L)\right)$; and

$\left(1_{\mu}\right)$ if $\mu \geqslant 2$ then $E_{1}(L) \subseteq \mathbf{Z} H$ is the product ideal $E_{1}(L)=\Delta_{1}(L) I H$, where $I H \subseteq \mathbf{Z H}$ is the ideal generated by $\left\{t_{1}-1, \ldots, t_{\mu}-1\right\}$.

(The ideal $I H$ may also be described as $I H=\operatorname{ker} \varepsilon$, where $\varepsilon: \mathbf{Z H} \rightarrow \mathbf{Z}$ is the homomorphism with $\varepsilon(h)=1 \forall h \in H$. This function $\varepsilon$ is known as the "augmentation map" or "trivializer" of $\mathbf{Z} H$, and $I H$ is the "augmentation ideal" of $\mathbf{Z} H$.)

In 1953, in [5], Guillermo Torres proved that if $\mu \geqslant 2$ then the Alexander polynomials of $L$ and its sublink $L_{\mu}=L-K_{\mu}$ satisfy:

Received by the editors March 26, 1980 and, in revised form, January 21, 1981.

1980 Mathematics Subject Classification. Primary 57M05, 57M25.

Key words and phrases. Tame links, elementary ideals, Alexander polynomials, Torres' relations.

(C) 1982 American Mathematical Society 0002-9947/82/0000-0776/\$05.50 
$\left(2_{2}\right)$ if $\mu=2$ then

$$
\left(t_{1}-1\right) \Delta_{1}(L)\left(t_{1}, 1\right)=\left(t_{1}^{l}-1\right) \Delta_{1}\left(L_{\mu}\right)\left(t_{1}\right),
$$

where $l$ is the linking number $l=l\left(K_{1}, K_{2}\right)$; and

$\left(2_{\mu}\right)$ if $\mu \geqslant 3$ then

$$
\Delta_{1}(L)\left(t_{1}, \ldots, t_{\mu-1}, 1\right)=\left(\left(\prod_{i<\mu} t_{i}^{l_{i}}\right)-1\right) \Delta_{1}\left(L_{\mu}\right)\left(t_{1}, \ldots, t_{\mu-1}\right),
$$

where if $1 \leqslant i<\mu, l_{i}$ is the linking number $l_{i}=l\left(K_{i}, K_{\mu}\right)$.

(These equations must be used with caution, since the Alexander polynomials are only defined within multiplication by units.)

An equivalent statement may be formulated as follows. If $G_{\mu}=\pi_{1}\left(S^{3}-L_{\mu}\right)$ and $H_{\mu}$ is its abelianization, then there is a (unique) homomorphism $\phi: \mathbf{Z} H \rightarrow \mathbf{Z} H_{\mu}$ with $\phi\left(t_{i}\right)=t_{i}$ whenever $1 \leqslant i<\mu$, and $\phi\left(t_{\mu}\right)=1$. Using the relations $\left(1_{\mu-1}\right)$ and $\left(1_{\mu}\right)$, the relations (2) can be reduced to the single property:

(2') if $\mu \geqslant 2$ then

$$
\phi E_{1}(L)=\left(\left(\prod_{i<\mu} t_{i}^{l_{i}}\right)-1\right) E_{1}\left(L_{\mu}\right) .
$$

Here we show that this generalizes to

THEOREM (1.1). In the situation just described,

$$
E_{k-1}\left(L_{\mu}\right)+\left(\left(\prod_{i<\mu} t_{i}^{l_{i}}\right)-1\right) E_{k}\left(L_{\mu}\right) \subseteq \phi E_{k}(L) \subseteq E_{k-1}\left(L_{\mu}\right)+I H_{\mu} \cdot E_{k}\left(L_{\mu}\right)
$$

for any $k \geqslant 1$.

Since $E_{0}(L)=0$ for any link, the first of these inclusions is the equality $\left(2^{\prime}\right)$ if $k=1$. The upper bound $E_{k-1}\left(L_{\mu}\right)+I H_{\mu} \cdot E_{k}\left(L_{\mu}\right)$ can be reduced somewhat by geometric considerations (see $\$ 4$ ).

It is, perhaps, unfortunate, but $\left(2^{\prime}\right)$ cannot be generalized to higher values of $k$ as an equality. Examples are given in $\$ 3$ which indicate that either of the inclusions of Theorem (1.1) may be an equality for a given link $L$ and value of $k$, or neither, or both.

Nineteen years before the publication of Torres' paper, Seifert had mentioned, in [4], that $\Delta_{1}(L)(1)=\varepsilon \Delta_{1}(L)= \pm 1$ if $\mu=1$. Torres used the relations (2) to deduce from this that $\Delta_{1}(L)(1,1)=\varepsilon \Delta_{1}(L)= \pm l$ if $\mu=2$, and $\Delta_{1}(L)(1, \ldots, 1)=\varepsilon \Delta_{1}(L)$ $=0$ if $\mu \geqslant 3$. Using the relations (1) and (2), and the fact that the elementary ideals of a tame link form an ascending sequence, one can actually verify these properties:

(i) if $\mu=1$ then $\varepsilon E_{k}(L)=\mathbf{Z}$ for any $k \geqslant 1$;

(ii) if $\mu=2$ then $E_{1}(L) \subseteq\left(\left(t_{1}-1\right)\left(t_{2}-1\right), t_{1}^{l}-1, t_{2}^{l}-1\right)$, where $l \in \mathbf{Z}$ is the linking number; and

(iii) if $\mu \geqslant 2$ then $E_{1}(L) \subseteq(I H)^{\mu-1}$.

We generalize these to:

(i') if $\mu \geqslant 1$ then $\varepsilon E_{k}(L)=\mathbf{Z}$ for any $k \geqslant \mu$; 
(ii') if $\mu \geqslant 2$ then $E_{\mu-1}(L) \subseteq J+C$, where $J \subseteq \mathbf{Z} H$ is the ideal generated by the products $\left(t_{p}-1\right)\left(t_{q}-1\right), p \neq q$, and $C \subseteq \mathbf{Z} H$ is the ideal generated by the elements $t_{p}^{l\left(K_{p}, K_{q}\right)}-1, p \neq q$; and

(iii') if $\mu \geqslant 2$ then $E_{k}(L) \subseteq(I H)^{\mu-k}$ for any $k<\mu$.

In particular, the augmented ideals $\varepsilon E_{k}(L)$ depend only on the number of components of $L: \varepsilon E_{k}(L)=0$ whenever $k<\mu$, and $\varepsilon E_{k}(L)=\mathbf{Z}$ whenever $k>\mu$.

This paper is a revised version of the second chapter of the author's Ph.D. thesis [6], which was written at Yale University under the guidance of William S. Massey, to whom we would like to express our deep gratitude. We are also grateful to the referee, who made several helpful suggestions. The author's wife, Sharon Richter, is to be thanked for drawing the illustrations.

2. The elementary ideals. In this section we outline a procedure by which the elementary ideals of a tame link can be obtained from any regular projection of it. The material is standard and appears, for instance, in Torres' paper [5].

Let $L \subseteq S^{3}$ be a tame link, given with a regular projection in the plane, which has been normalized by removing short arcs surrounding the underpassing point of each crossing. We denote the arc components of the projection $e_{i j}(1 \leqslant i \leqslant \mu$ and $1 \leqslant j \leqslant j_{i}$ ); they are indexed so that for each $i, e_{i 1} \cup \cdots \cup e_{i j_{i}}$ is the image of $K_{i}$ in the projection and $e_{i 1}, \ldots, e_{i j_{i}}$ appear consecutively around $K_{i}$, determining an orientation of it. We call the crossing that separates $e_{i j}$ from $e_{i j+1}$ the $i j$ th crossing of the projection, and say it is of type +1 or -1 according to whether the overpassing arc is oriented from left to right or from right to left, relative to the orientation of the underpassing component. If $i \neq j \in\{1, \ldots, \mu\}$ the linking number $l\left(K_{i}, K_{j}\right)$ is the sum of the types of the crossings of $K_{i}$ over $K_{j}[3$, p. 132].

A presentation $\left\langle x_{i j} ; r_{i j}\right\rangle$ of $G=\pi_{1}\left(S^{3}-L\right)$ is associated to this projection. A generator $x_{i j}$ corresponds to each arc $e_{i j}$, and a relator $r_{i j}$ corresponds to each crossing; if $e_{p q}$ is the overpassing arc of the $i j$ th crossing, $r_{i j}$ is $x_{p q} x_{i j} x_{p q}^{-1} x_{i j+1}^{-1}$ or $x_{p q}^{-1} x_{i j} x_{p q} x_{i j+1}^{-1}$, according to whether the crossing is of type +1 or -1 . If $F$ is the free group on $\left\{x_{i j}\right\}, \psi: F \rightarrow G$ is the epimorphism associated to the presentation, and $\alpha: G \rightarrow H=G /[G, G]$ is the abelianizing homomorphism, then $\alpha \psi\left(x_{i j}\right)=$ $\alpha \psi\left(x_{i k}\right) \forall i \forall j, k \in\left\{1, \ldots, j_{i}\right\}$, and $\left\{t_{i}=\alpha \psi\left(x_{i 1}\right)\right\}$ freely generates $H$.

If $\left\langle x_{1}, \ldots, x_{n} ; r_{1}, \ldots, r_{m}\right\rangle$ is finite presentation of the group $G$, and $\bar{F}$ is the free group on $\left\{x_{1}, \ldots, x_{n}\right\}$, then there is a surjection $\bar{\psi}: \bar{F} \rightarrow G$ whose kernel is the normal subgroup of $\bar{F}$ generated by $\left\{r_{1}, \ldots, r_{m}\right\}$. The Alexander matrix of this presentation is the $m \times n$ matrix, with entries in $\mathbf{Z} H$, whose $i j$ th entry is $a \bar{\psi}\left(\partial\left(r_{i}\right) / \partial x_{j}\right)$ whenever $1 \leqslant i \leqslant m$ and $1 \leqslant j \leqslant n$. The elementary ideals $E_{k}(G)$, $k \geqslant 0$, are defined by: $E_{k}(G)=0$ whenever $k<n-m, E_{k}(G)=\mathbf{Z} H$ whenever $k \geqslant n$, and if $n-m \leqslant k<n$ then $E_{k}(G) \subseteq \mathbf{Z} H$ is the ideal generated by the determinants of the $(n-k) \times(n-k)$ submatrices of the Alexander matrix. (These ideals do not depend on the particular presentation of $G$ used to construct the Alexander matrix, as shown in [1, pp. 104-107].) The elementary ideals $E_{k}(L)$ of a link $L$ are those of its group.

It is well known that any one of the relators $r_{i j}$ is a consequence of the others (see [3, p. 60]). Since there are at least as many generators $x_{i j}$ as relators $r_{i j}$ in the 
presentation, this shows that any tame link $L$ possesses an Alexander matrix with strictly fewer rows than columns, and hence $E_{0}(L)=0$.

3. Examples. In this section the elementary ideals of certain links are calculated, using the procedure of $\$ 2$. To facilitate the calculations, the group presentations have been simplified prior to the derivation of the associated Alexander matrix. Also, if the final version of a presentation has as many relators as generators, then the final relator is simply ignored in the calculation of the Alexander matrix.

In each of the first five examples, sufficiently many of the elementary ideals are given to determine all of them, using the facts that $E_{0} \equiv 0$ and the elementary ideals of a tame link form an ascending sequence.

The links of Examples 3 and 5 appear in the table at the end of [3] as $4{ }_{1}^{2}$ and $6_{2}^{3}$, respectively. Two projections are provided of the latter, only one of which is used in the calculation of the ideals.

EXAmple 1. The trivial link $T^{\mu}$ of $\mu$ components. $G \cong\left\langle x_{11}, \ldots, x_{\mu 1} ; 1\right\rangle$. The Alexander matrix of this presentation is the $1 \times \mu$ matrix $\left(\begin{array}{lll}0 & 0 \cdots 0\end{array}\right)$. The elementary ideals are $E_{\mu}=\mathbf{Z} H$, and $E_{\mu-1}=0$.

EXAMPLE 2. (Note the ordering of the components.)

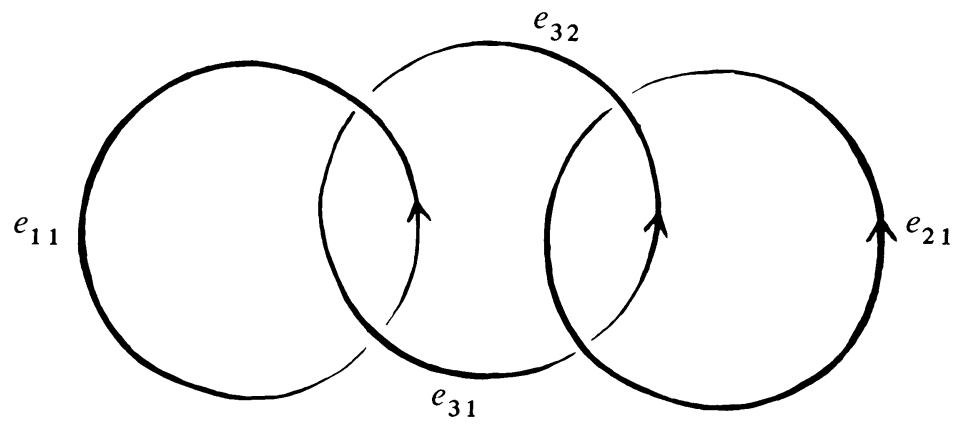

$G \cong\left\langle x_{11}, x_{21}, x_{31} ; x_{31} x_{11} x_{31}^{-1} x_{11}^{-1}, x_{31} x_{21} x_{31}^{-1} x_{21}^{-1}\right\rangle$. The Alexander matrix of this presentation is

$$
\left(\begin{array}{ccc}
t_{3}-1 & 0 & 1-t_{1} \\
0 & t_{3}-1 & 1-t_{2}
\end{array}\right) .
$$

The elementary ideals are $E_{1}=\left(t_{3}-1\right) I H, E_{2}=I H$, and $E_{3}=\mathbf{Z} H$.

ExAMPLE 3. The link 4 .

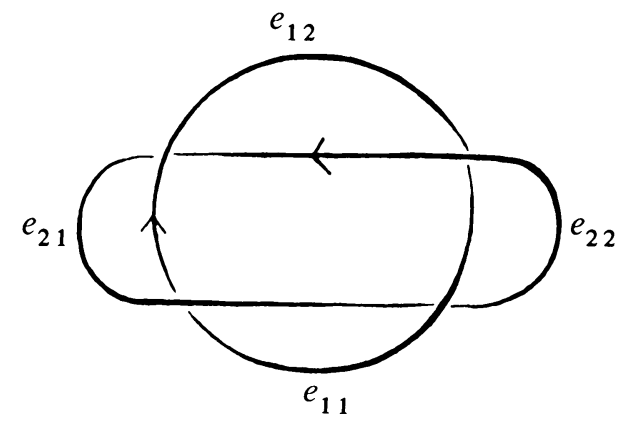

$$
G \cong\left\langle x_{11}, x_{21} ; x_{11}^{-1} x_{21}^{-1} x_{11} x_{21}^{-1} x_{11} x_{21} x_{11}^{-1} x_{21}, x_{21}^{-1} x_{11}^{-1} x_{21} x_{11}^{-1} x_{21} x_{11} x_{21}^{-1} x_{11}\right\rangle
$$


The Alexander matrix of this presentation is

$$
\left(\left(t_{2}^{-1}-1\right)\left(t_{1}^{-1}+t_{2}^{-1}\right)\left(t_{1}-1\right)\left(t_{2}^{-2}+t_{1}^{-1} t_{2}^{-1}\right)\right) \text {. }
$$

The elementary ideals are $E_{1}=\left(t_{1}^{-1}+t_{2}^{-1}\right) I H=\left(t_{1}+t_{2}\right) I H$, and $E_{2}=\mathbf{Z} H$.

EXAMPLE 4.

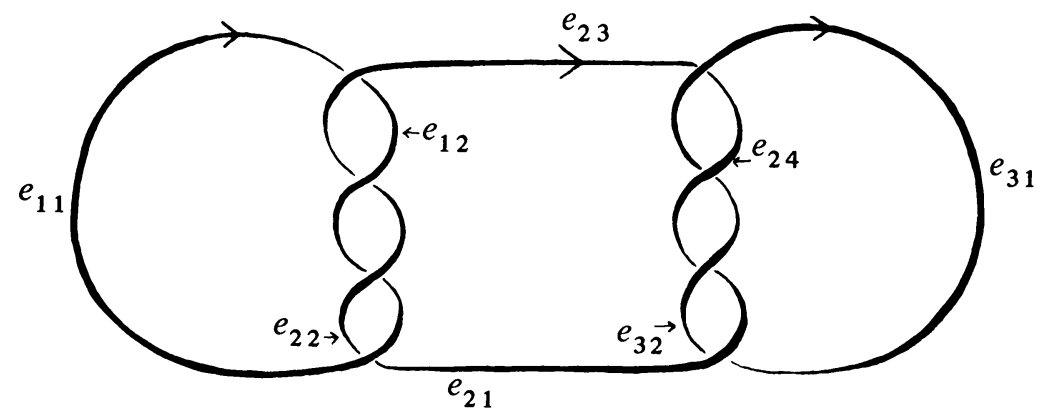

$$
\begin{aligned}
G \cong\left\langle x_{11}, x_{21}, x_{31} ; x_{21}^{-1} x_{11} x_{21} x_{11}^{-1} x_{21} x_{11}^{-1} x_{21}^{-1} x_{11},\right. \\
\left.\quad x_{21}^{-1} x_{31}^{-1} x_{21} x_{31}^{-1} x_{21} x_{31} x_{21}^{-1} x_{31}, x_{21}^{-1} x_{31} x_{21} x_{31}^{-1} x_{21} x_{31}^{-1} x_{21}^{-1} x_{31}\right\rangle .
\end{aligned}
$$

The Alexander matrix of this presentation is

$$
\left(\begin{array}{ccc}
\left(t_{1}^{-1}+t_{2}^{-1}\right)\left(1-t_{2}\right) & \left(t_{1}^{-1}+t_{2}^{-1}\right)\left(t_{1}-1\right) & 0 \\
0 & \left(t_{3}^{-1}-1\right)\left(t_{2}^{-1}+t_{3}^{-1}\right) & \left(t_{2}-1\right)\left(t_{3}^{-2}+t_{2}^{-1} t_{3}^{-1}\right)
\end{array}\right)
$$

The elementary ideals are $E_{1}=\left(t_{2}-1\right)\left(t_{1}+t_{2}\right)\left(t_{2}+t_{3}\right) I H$ $E_{2}=\left(\left(t_{1}-1\right)\left(t_{1}+t_{2}\right),\left(t_{2}-1\right)\left(t_{1}+t_{2}\right),\left(t_{2}-1\right)\left(t_{2}+t_{3}\right),\left(t_{3}-1\right)\left(t_{2}+t_{3}\right)\right)$, and $E_{3}=\mathbf{Z} H$.

EXAMPLE 5. The Borromean rings (the link $6_{2}^{3}$ ).

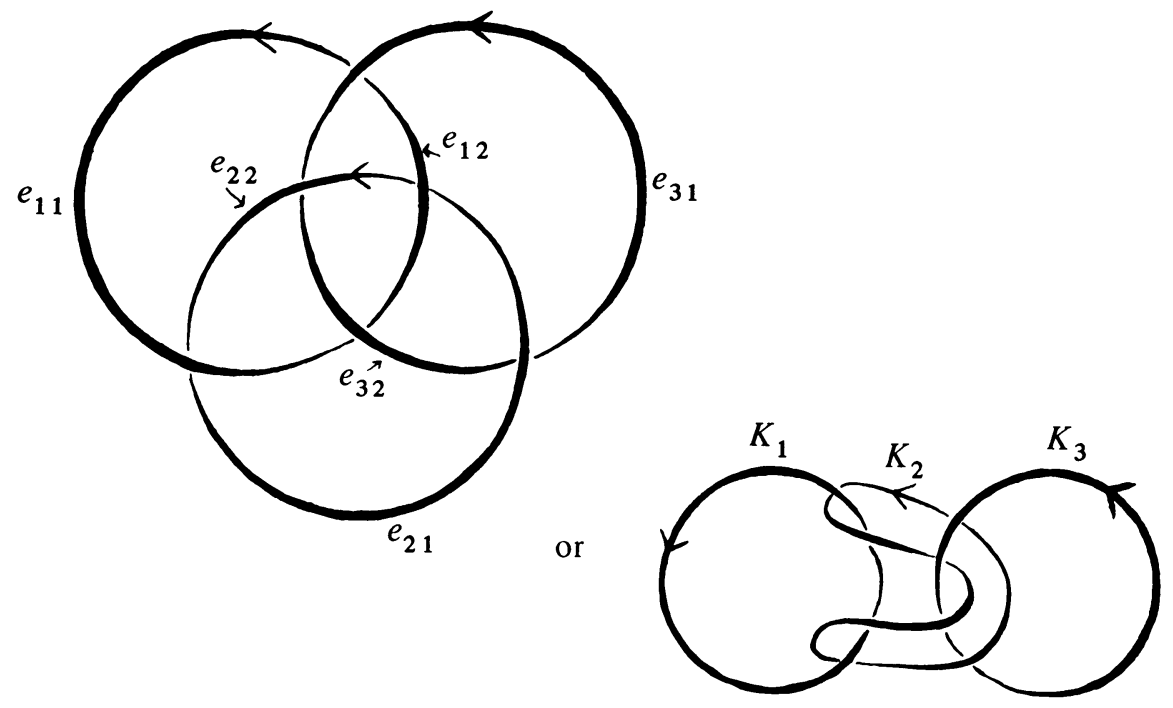

$$
\begin{aligned}
G \cong\left\langle x_{11}, x_{21},\right. & x_{31} ; x_{21} x_{31} x_{21}^{-1} x_{11} x_{21} x_{31}^{-1} x_{21}^{-1} x_{31} x_{11}^{-1} x_{31}^{-1}, \\
& \left.x_{31} x_{11} x_{31}^{-1} x_{21} x_{31} x_{11}^{-1} x_{31}^{-1} x_{11} x_{21}^{-1} x_{11}^{-1}, x_{11} x_{21} x_{11}^{-1} x_{31} x_{11} x_{21}^{-1} x_{11}^{-1} x_{21} x_{31}^{-1} x_{21}^{-1}\right\rangle .
\end{aligned}
$$


The Alexander matrix of this presentation is

$$
\left(\begin{array}{ccc}
0 & \left(1-t_{1}\right)\left(1-t_{3}\right) & \left(t_{1}-1\right)\left(1-t_{2}\right) \\
\left(t_{2}-1\right)\left(1-t_{3}\right) & 0 & \left(t_{1}-1\right)\left(t_{2}-1\right)
\end{array}\right) .
$$

The elementary ideals are

$$
\begin{aligned}
& E_{1}=\left(t_{1}-1\right)\left(t_{2}-1\right)\left(t_{3}-1\right) I H, \\
& E_{2}=\left(\left(t_{1}-1\right)\left(t_{2}-1\right),\left(t_{2}-1\right)\left(t_{3}-1\right),\left(t_{1}-1\right)\left(t_{3}-1\right)\right), \text { and } \\
& E_{3}=\mathbf{Z} H .
\end{aligned}
$$

EXAMPLE 6. The simple chain $C^{\mu}$ of $\mu$ components.

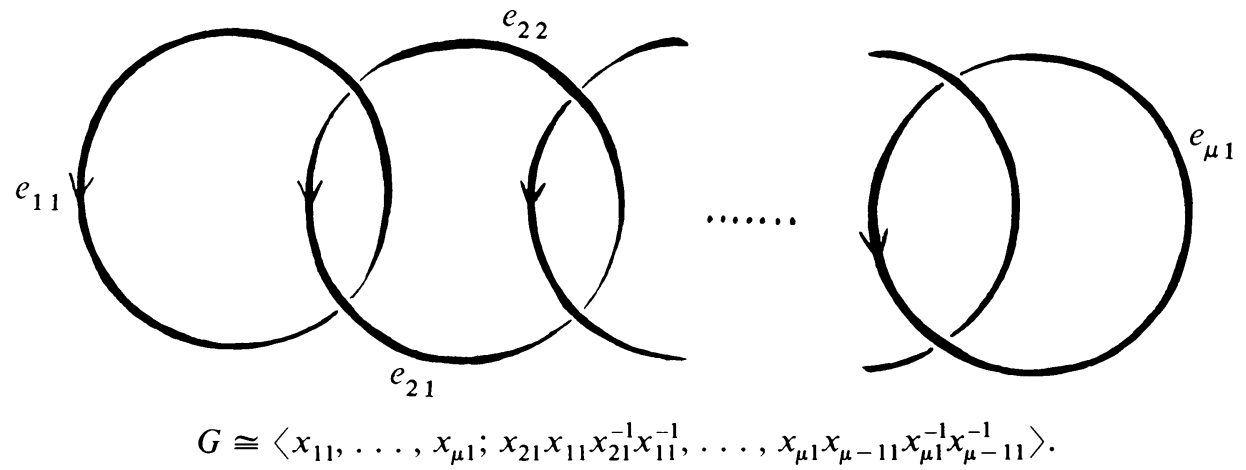

The Alexander matrix of this presentation is

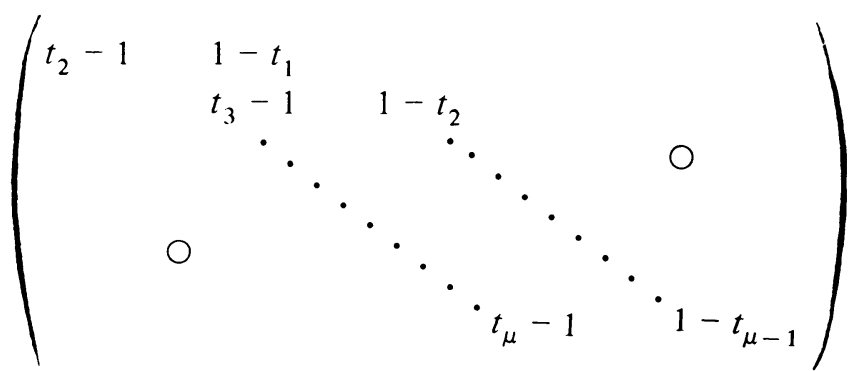

We observe only that $E_{\mu}=\mathbf{Z} H$ and $E_{\mu-1}=I H$.

Consider the link $L$ of Example 2. Its sublink $L_{\mu}$ is the trivial link $T^{2}$ of two components (Example 1), so $E_{1}\left(L_{\mu}\right)=0$ and $E_{2}\left(L_{\mu}\right)=\mathbf{Z} H_{\mu}$. Hence

$$
E_{1}\left(L_{\mu}\right)+\left(\left(\prod_{i<\mu} t_{i}^{l_{i}}\right)-1\right) E_{2}\left(L_{\mu}\right)=0+\left(\left(t_{1} t_{2}\right)-1\right) \mathbf{Z} H_{\mu}
$$

is the principal ideal of $\mathbf{Z} H_{\mu}$ generated by $\left(t_{1} t_{2}\right)-1$, and

$$
E_{1}\left(L_{\mu}\right)+I H_{\mu} \cdot E_{2}\left(L_{\mu}\right)=0+I H_{\mu} \cdot \mathbf{Z} H_{\mu}=I H_{\mu} .
$$

Since $E_{2}(L)=I H, \phi E_{2}(L)=I H_{\mu}=E_{1}\left(L_{\mu}\right)+I H_{\mu} \cdot E_{2}\left(L_{\mu}\right)$. This shows that the second inclusion of Theorem (1.1) may be an equality when the first is not.

If we consider instead the link $L$ of Example 4, then its sublink $L_{\mu}$ is the link of Example 3, so $E_{1}\left(L_{\mu}\right)=\left(t_{1}+t_{2}\right) I H_{\mu}$, and $E_{2}\left(L_{\mu}\right)=\mathbf{Z} H_{\mu}$. Hence

$$
\begin{aligned}
E_{1}\left(L_{\mu}\right)+\left(\left(\prod_{i<\mu} t_{i}^{t^{\prime}}\right)-1\right) & E_{2}\left(L_{\mu}\right)=\left(t_{1}+t_{2}\right) I H_{\mu}+\left(t_{2}^{2}-1\right) \mathbf{Z} H_{\mu} \\
= & \left(\left(t_{1}+t_{2}\right)\left(t_{1}-1\right),\left(t_{1}+t_{2}\right)\left(t_{2}-1\right),\left(t_{2}+1\right)\left(t_{2}-1\right)\right),
\end{aligned}
$$


and

$$
E_{1}\left(L_{\mu}\right)+I H_{\mu} \cdot E_{2}\left(L_{\mu}\right)=I H_{\mu} \cdot \mathbf{Z} H_{\mu}=I H_{\mu}
$$

Thus

$$
\begin{aligned}
\phi E_{2}(L) & =\left(\left(t_{1}-1\right)\left(t_{1}+t_{2}\right),\left(t_{2}-1\right)\left(t_{1}+t_{2}\right),\left(t_{2}-1\right)\left(t_{2}+1\right), 0\right) \\
& =E_{1}\left(L_{\mu}\right)+\left(\left(\prod_{i<\mu} t_{i}^{t_{i}}\right)-1\right) E_{2}\left(L_{\mu}\right) .
\end{aligned}
$$

This shows that the first inclusion of Theorem (1.1) may be an equality when the second is not.

The link of Example 5 shows that it is possible for neither of the inclusions of Theorem (1.1) to be an equality $(k=2)$, and the links of Example 6 show that it is possible for both inclusions to be equalities at the same time $(k=\mu-1)$.

The inclusions of (ii') and (iii') are realized as equalities by the $E_{\mu-1}$ of Examples 2 and 6; the inclusion of (ii') is also an equality for Example 5. According to calculations not presented here, (ii') is an equality for twenty-seven more of the three-component links listed in Appendix $C$ of [3], all but $8_{4}^{3}, 8_{6}^{3}, 8_{10}^{3}, 9_{11}^{3}, 9_{12}^{3}, 9_{20}^{3}$, and $9_{21}^{3}$.

4. Proof of Theorem (1.1). Suppose that a tame link $L=K_{1} \cup \cdots \cup K_{\mu} \subseteq S^{3}$ of $\mu \geqslant 2$ components is given, together with a regular projection in the plane. The projection is assumed to contain no crossings in which the overpassing arc is one of the underpassing arcs. (We call such crossings "trivial"; clearly they can be adjoined to, or deleted from, a link's projection at will.) It is also convenient to assume that there are as many crossings in which $K_{\mu}$ is the underpassing component as there are arcs $e_{\mu j}$ in the projection. (If this is not the case, then $K_{\mu}$ is an unknotted component of $L$ which is unlinked from the rest of $L$, and the situation can be remedied by "sliding it under" some other component of $L$ to introduce nontrivial crossings over $K_{\mu}$.)

Following the procedure of $\S 2$, a presentation $\left\langle x_{i j} ; r_{i j}\right\rangle$ of $G=\pi_{1}\left(S^{3}-L\right)$ is obtained, in which the generators $x_{i j}$ are in one-to-one correspondence with the arcs $e_{i j}$ and the relators $r_{i j}$ are in one-to-one correspondence with the crossings of the projection. If this presentation is used in the construction of the Alexander matrix, then an $m \times n$ (where $m$ is the number of crossings of the projection, and $n=\sum_{i=1}^{\mu} j_{i}$ is the number of arcs $e_{i j}$ ) matrix is obtained. ${ }^{1}$ Each row of the matrix has precisely three nonzero entries; explicitly, the row corresponding to the $i j$ th crossing has these nonzero entries: $t_{p}$ or $t_{p}^{-1}$ in the column corresponding to $e_{i j}$ (according to whether the crossing is of type +1 or -1 ), -1 in the column corresponding to $e_{i j+1}$ (independent of the type of the crossing), and $1-t_{i}$ or $t_{p}^{-1}\left(t_{i}-1\right)$ in the column corresponding to the overpassing arc $e_{p q}$ (according to whether the crossing is of type +1 or -1 ).

\footnotetext{
${ }^{1}$ Unless $L$ has some unknotted component unlinked from the rest of $L, m=n$, and the Alexander matrix is square. (In fact, even if $L$ has such a component, it will still have some regular projections for which $m=n$.) Eliminating any redundant row(s), though convenient when dealing with specific examples, does not simplify the ensuing arguments at all, so we do not eliminate any rows.
} 
The rows and columns of this matrix may be partitioned as indicated in Figure 1.

$\left.\begin{array}{l|c}\begin{array}{l}\text { rows corresponding to } \\ i j \text { th crossings, } i<\mu\end{array} \\ \begin{array}{l}\text { rows corresponding to } \\ \mu j \text { th crossings }\end{array} \\ \text { to } \operatorname{arcs} e_{i j}, i<\mu\end{array}\right)=M$

Figure 1

Let $M^{\prime}, N, D$, and $D^{\prime}$ be the indicated submatrices of the Alexander matrix $M$.

An obvious property of determinants is their functoriality, that is, if $P$ is a square matrix with entries in some commutative ring $R, f: R \rightarrow R^{\prime}$ is a homomorphism, and $f(P)$ is the matrix whose entries are the images under $f$ of the entries of $P$, then $\operatorname{det} f(P)=f(\operatorname{det} P)$.

Using the definition of the elementary ideals $E_{k}(L)=E_{k}(G)$, it follows that $\phi E_{k}(L)=0$ whenever $0 \leqslant k<n-m, \phi E_{k}(L)=\mathbf{Z} H_{\mu}$ whenever $k \geqslant n$, and if $n-m \leqslant k<n$ then $\phi E_{k}(L)$ is the ideal of $\mathbf{Z} H_{\mu}$ generated by the determinants of the $(n-k) \times(n-k)$ submatrices of $\phi(M)$.

Torres notes in [5] that $\phi\left(M^{\prime}\right)$ is an Alexander matrix for the link $L_{\mu}=$ $K_{1} \cup \cdots \cup K_{\mu-1}$; in fact it is easy to see that a regular projection of $L_{\mu}$ which yields this Alexander matrix can be obtained from the original projection of $L$ by replacing each crossing in which $K_{\mu}$ passes over some other component of $L$ by a trivial crossing. (See Figure 2.)
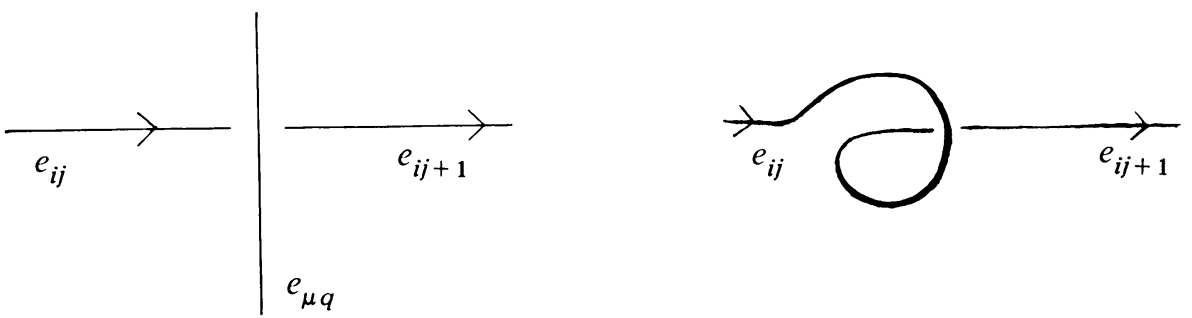

Original crossing is replaced by a trivial crossing.

Figure 2

Note that the same trivial crossing is inserted, irrespective of the type of the original crossing.

By hypothesis, there are as many crossings in which $K_{\mu}$ is the undercrossing component as there are arcs $e_{\mu j}$, that is, $D$ is a $j_{\mu} \times j_{\mu}$ matrix. Hence $\phi\left(M^{\prime}\right)$ is an $\left(m-j_{\mu}\right) \times\left(n-j_{\mu}\right)$ Alexander matrix for $L_{\mu}$. By the definition of $\$ 2$, it follows that $E_{k}\left(L_{\mu}\right)=0$ whenever $0 \leqslant k<\left(n-j_{\mu}\right)-\left(m-j_{\mu}\right)=n-m, \quad E_{k}\left(L_{\mu}\right)=\mathbf{Z} H_{\mu}$ whenever $k \geqslant n$. $-j_{\mu}$, and if $n-m \leqslant k<n-j_{\mu}$, then $E_{k}\left(L_{\mu}\right)$ is the ideal of $\mathbf{Z} H_{\mu}$ generated by the determinants of the $\left(n-j_{\mu}-k\right) \times\left(n-j_{\mu}-k\right)$ submatrices of $\phi\left(M^{\prime}\right)$. 
In particular, $\phi E_{k}(L)=E_{k}\left(L_{\mu}\right)=E_{k-1}\left(L_{\mu}\right)=0$ whenever $0 \leqslant k<n-m$, and $\phi E_{k}(L)=E_{k}\left(L_{\mu}\right)=E_{k-1}\left(L_{\mu}\right)=\mathbf{Z} H_{\mu}$ whenever $k \geqslant n$, so both inclusions of Theorem (1.1) are equalities if $k<n-m$ or $k \geqslant n$.

Consider the matrix $\phi(D)$; the description, at the beginning of this section, of the entries of $M$ leads immediately to the conclusion that

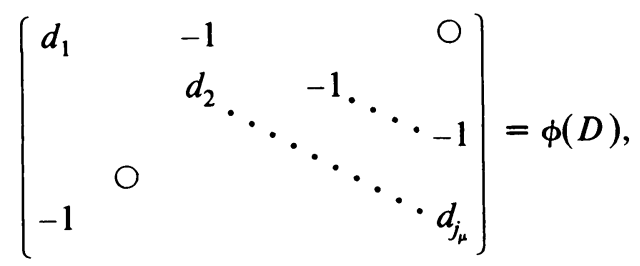

where if $1 \leqslant j \leqslant j_{\mu}$ the diagonal entry $d_{j}$ is 1 , $t_{p}$, or $t_{p}^{-1}$, according to whether $K_{\mu}$ passes over itself in the $\mu j$ th crossing, or $1 \leqslant p<\mu$ and $K_{p}$ passes over $K_{\mu}$ in the $\mu j$ th crossing, which is of type +1 , or $1 \leqslant p<\mu$ and $K_{p}$ passes over $K_{\mu}$ in the $\mu$ jth crossing, which is of type -1 . As Torres notes in [5], it follows that

$$
\operatorname{det} \phi(D)=\left(\prod_{j=1}^{j_{\mu}} d_{j}\right)-1=\left(\prod_{i=1}^{\mu-1} t_{i}^{i}\right)-1,
$$

where $l_{i}=l\left(K_{i}, K_{\mu}\right)$ whenever $1 \leqslant i<\mu$. Furthermore, if $D_{0}$ is the submatrix of $D$ obtained by deleting its first column and last row, clearly $\operatorname{det} \phi\left(D_{0}\right)= \pm 1$.

In particular, $\phi(M)$ has the $\left(j_{\mu}-1\right) \times\left(j_{\mu}-1\right)$ submatrix $\phi\left(D_{0}\right)$ with $\operatorname{det} \phi\left(D_{0}\right)$ $= \pm 1$, and hence $\phi E_{n-j_{\mu}+1}(L)=\mathbf{Z} H_{\mu}$. Since the ideals of $L$ form an ascending sequence, it follows that $\phi E_{k}(L)=\mathbf{Z} H_{\mu}$ whenever $k>n-j_{\mu}$, and hence $\phi E_{k}(L)=$ $E_{k-1}\left(L_{\mu}\right)=\mathbf{Z} H_{\mu}$ whenever $k>n-j_{\mu}$. Thus both inclusions of Theorem (1.1) are equalities if $k>n-j_{\mu}$.

It remains to consider the values $n-m \leqslant k \leqslant n-j_{\mu}$.

Torres notes in [5] that $\phi\left(D^{\prime}\right)=0$; this follows also from the description of the entries of $M$ given at the beginning of this section. Thus if $1 \leqslant p \leqslant m-j_{\mu}$, and $P$ is a $p \times p$ submatrix of $\phi\left(M^{\prime}\right), \phi(M)$ has a $\left(p+j_{\mu}-1\right) \times\left(p+j_{\mu}-1\right)$ submatrix

$$
\left(\begin{array}{cc}
P & X \\
0 & \phi\left(D_{0}\right)
\end{array}\right) \quad \text { with } \operatorname{det}\left(\begin{array}{cc}
P & X \\
0 & \phi\left(D_{0}\right)
\end{array}\right)= \pm \operatorname{det} P
$$

and $\phi(M)$ also has a $\left(p+j_{\mu}\right) \times\left(p+j_{\mu}\right)$ submatrix $\left(\begin{array}{c}P \\ 0\end{array} \underset{\phi(D)}{Y}\right)$ with

$$
\operatorname{det}\left(\begin{array}{cc}
P & Y \\
0 & \phi(D)
\end{array}\right)=(\operatorname{det} \phi(D))(\operatorname{det} P)=\left(\left(\prod_{i=1}^{\mu-1} t_{i}^{t_{i}}\right)-1\right) \operatorname{det} P .
$$

(Here $X$ and $Y$ are submatrices of $\phi(N)$ which do not affect the values of the determinants of the matrices in which they appear.) It follows that $E_{k-1}\left(L_{\mu}\right) \subseteq$ $\phi E_{k}(L)$ whenever $n-m<k \leqslant n-j_{\mu}$, and $\left(\left(\prod_{i=1}^{\mu-1} t_{i}^{t_{i}}\right)-1\right) E_{k}\left(L_{\mu}\right) \subseteq \phi E_{k}(L)$ whenever $n-m \leqslant k<n-j_{\mu}$. Certainly $E_{n-m-1}\left(L_{\mu}\right)=0 \subseteq \phi E_{n-m}(L)$. Also, since $\phi(D)$ is a $j_{\mu} \times j_{\mu}$ submatrix of $\phi(M)$, the principal ideal

$$
(\operatorname{det} \phi(D))=\left(\left(\prod_{i<\mu} t_{i}^{i_{i}}\right)-1\right) \cdot \mathbf{Z} H_{\mu}=\left(\left(\prod_{i<\mu} t_{i}^{l_{i}}\right)-1\right) E_{n-j_{\mu}}\left(L_{\mu}\right)
$$


is contained in $\phi E_{n-j_{\mu}}(L)$. Thus

$$
E_{k-1}\left(L_{\mu}\right)+\left(\left(\prod_{i<\mu} t_{i}^{l_{i}}\right)-1\right) E_{k}\left(L_{\mu}\right) \subseteq \phi E_{k}(L)
$$

whenever $n-m \leqslant k \leqslant n-j_{\mu}$.

To verify the second inclusion of Theorem (1.1), it must be shown that $\operatorname{det} X \in$ $E_{k-1}\left(L_{\mu}\right)+I H_{\mu} \cdot E_{k}\left(L_{\mu}\right)$ whenever $n-m \leqslant k<n-j_{\mu}$ and $X$ is an $(n-k) \times$ $(n-k)$ submatrix of $\phi(M) .^{2}$ The argument is split into three cases.

Case 1. $X$ does not involve some of the last $j_{\mu}$ columns of $\phi(M)$. Suppose that $X$ involves precisely $j$ of the last $j_{\mu}$ columns of $\phi(M), j<j_{\mu}$. Expanding $\operatorname{det} X$ by minors along the last $j$ columns of $X$ yields an expression of $\operatorname{det} X$ as a sum in which each summand is a multiple of the determinant of some $(n-k-j) \times$ $(n-k-j)$ submatrix of $\phi\left(M^{\prime}\right)$. Thus det $X$ can be expressed as a sum of certain elements of $E_{k+j-j_{\mu}}\left(L_{\mu}\right)$, so det $X \in E_{k+j-j_{\mu}}\left(L_{\mu}\right)$. Since $j<j_{\mu}, k+j-j_{\mu} \leqslant k-1$, and hence $E_{k+j-j_{\mu}}\left(L_{\mu}\right) \subseteq E_{k-1}\left(L_{\mu}\right)$, since the ideals of $L_{\mu}$ form an ascending sequence. Thus det $X \in E_{k-1}\left(L_{\mu}\right)$ in this case.

Case 2. $X$ involves all of the last $j_{\mu}$ rows and columns of $\phi(M)$. Then $X$ is of the form $X=\left(\begin{array}{cc}\bar{X} & Y \\ 0 & \phi(D)\end{array}\right)$ for some $\left(n-k-j_{\mu}\right) \times\left(n-k-j_{\mu}\right)$ submatrix $\bar{X}$ of $\phi\left(M^{\prime}\right)$ and $\left(n-k-j_{\mu}\right) \times j_{\mu}$ submatrix $Y$ of $\phi(N)$, and hence

$$
\operatorname{det} X=(\operatorname{det} \phi(D))(\operatorname{det} \bar{X})=\left(\left(\prod_{i<\mu} t_{i}^{l_{i}}\right)-1\right) \operatorname{det} \bar{X} \in\left(\left(\prod_{i<\mu} t_{i}^{l_{i}}\right)-1\right) E_{k}\left(L_{\mu}\right) \text {. }
$$

Clearly

$$
\bar{\varepsilon}\left(\left(\prod_{i<\mu} t_{i}^{l_{i}}\right)-1\right)=\prod_{i<\mu} 1^{l_{i}}-1=1-1=0
$$

so

$$
\left(\left(\prod_{i<\mu} t_{i}^{l_{i}}\right)-1\right) \in \operatorname{ker} \bar{\varepsilon}=I H_{\mu} .
$$

(Here $\bar{\varepsilon}: \mathbf{Z} H_{\mu} \rightarrow \mathbf{Z}$ is the augmentation $\bar{\varepsilon}(h)=1 \forall h \in H_{\mu}$.) Thus $\operatorname{det} X \in I H_{\mu}$. $E_{k}\left(L_{\mu}\right)$ in this case.

Case 3. $X$ involves all of the last $j_{\mu}$ columns of $\phi(M)$, but does not involve some of the last $j_{\mu}$ rows of $\phi(M)$. Expanding det $X$ by minors along the last $j_{\mu}$ columns of $X$ yields an expression of $\operatorname{det} X$ as a sum in which each summand is some multiple of a product $e d$, where $e$ is some entry of $\phi(N)$ and $d$ is the determinant of some $\left(n-k-j_{\mu}\right) \times\left(n-k-j_{\mu}\right)$ submatrix of $\phi\left(M^{\prime}\right)$. From the description of the entries of $M$ given at the beginning of the section, it can easily be deduced that every nonzero entry of $\phi(N)$ is $\pm\left(t_{i}-1\right)$ for some $i \in\{1, \ldots, \mu-1\}$, i.e., every entry of $\phi(N)$ is in $I H_{\mu}$. Thus det $X$ can be expressed as a sum in which each summand is in $I H_{\mu} \cdot E_{k}\left(L_{\mu}\right)$, so det $X \in I H_{\mu} \cdot E_{k}\left(L_{\mu}\right)$ in this case. Q.E.D.

Certain geometric considerations may be used to limit the upper bound of Theorem (1.1) significantly. Let $\pi:\left(S^{3}-\{p t\}\right) \rightarrow \mathbf{R}^{2}$ be an orthogonal projection map which, when restricted to $L$, yields the given regular projection of $L$ in the

\footnotetext{
${ }^{2}$ The second inclusion is trivially true if $k=n-j_{\mu}$, for $\phi\left(M^{\prime}\right)$ certainly has entries which are units of $\mathbf{Z} H_{\mu}$, so $E_{n-j_{\mu}-1}\left(L_{\mu}\right)=\mathbf{Z} H_{\mu}$.
} 
plane. Let $U_{\mu}^{\pi}=\left\{i \mid 1 \leqslant i<\mu\right.$ and there is a crossing in $\pi(L)$ in which $K_{i}$ passes under $\left.K_{\mu}\right\}$, and let $I_{\mu}^{\pi}$ be the ideal of $\mathbf{Z} H_{\mu}$ generated by $\left\{t_{i}-1 \mid i \in U_{\mu}^{\pi}\right\}$. Then

LEMMA (4.1). The second inclusion of Theorem (1.1) still holds if $E_{k-1}\left(L_{\mu}\right)+$ $I H_{\mu} \cdot E_{k}\left(L_{\mu}\right)$ is replaced by $E_{k-1}\left(L_{\mu}\right)+I_{\mu}^{\pi} \cdot E_{k}\left(L_{\mu}\right)$.

Proof. If $1 \leqslant i<\mu$ and $l_{i} \neq 0$ then $K_{i}$ must pass under $K_{\mu}$ in some crossing of any regular projection of $L$, since $l_{i}=l\left(K_{i}, K_{\mu}\right)=l\left(K_{\mu}, K_{i}\right)$ can be calculated by considering only such crossings, as noted in $\$ 2$. Hence $i \in U_{\mu}^{\pi}$ whenever $1<i<\mu$ and $l_{i} \neq 0$, so $\left(t_{i}-1\right) \in I_{\mu}^{\pi}$ whenever $1 \leqslant i<\mu$ and $l_{i} \neq 0$. The element $\left(\Pi_{i<\mu} t_{i}^{l_{i}}\right)$ - 1 of $\mathrm{ZH}_{\mu}$ can be expressed as

$$
\left(\prod_{i<\mu} t_{i}^{l_{i}}\right)-1=\sum_{l_{i} \neq 0}\left(t_{i}-1\right) y_{i}
$$

where $y_{i}$ is

$$
\left(\prod_{k<i} t_{k}^{l_{k}}\right)\left(\sum_{j=1}^{l_{i}} t_{i}^{j-1}\right) \text { or }-\left(\prod_{k<i} t_{k}^{l_{k}}\right)\left(\sum_{j=-1}^{l_{i}} t_{i}^{j}\right)
$$

according to whether $l_{i}>0$ or $l_{i}<0$. Since $\left(t_{i}-1\right) \in I_{\mu}^{\pi}$ whenever $l_{i} \neq 0$, this shows that $\left(\Pi_{i<\mu} t_{i}^{l_{i}}\right)-1 \in I_{\mu}^{\pi}$.

Furthermore, if $N$ is the submatrix of the Alexander matrix $M$ indicated in Figure 1 , then every entry of $\phi(N)$ is an element of $I_{\mu}^{\pi}$, as can be deduced from the description of the entries of $M$ given at the beginning of this section. Q.E.D.

A simple application of this lemma is

COROLlaRY (4.2). Suppose that L has a projection $\pi(L)$ such that $U_{\mu}^{\pi}$ contains only a single element $u_{0}$, and suppose further that $l\left(K_{u_{0}}, K_{\mu}\right)= \pm 1$. Then the first inclusion of Theorem (1.1) is an equality for any $k \geqslant 1$.

PROof. For then the lower bound of Theorem (1.1) coincides with $E_{k-1}\left(L_{\mu}\right)+I_{\mu}^{\pi}$ $\cdot E_{k}\left(L_{\mu}\right)$.

In particular, any link $L=K_{1} \cup K_{2}$ with $l\left(K_{1}, K_{2}\right)= \pm 1$ satisfies the hypothesis of this corollary. (Note that in this case the upper bounds provided by Theorem (1.1) and the lemma are identical.)

Another way of using the lemma is by considering the various upper bounds it gives when the projection $\pi$ is varied. For instance, consider the link $L$ of Example 5 in $\S 3$. As shown there, $L$ has projections $\pi$ and $\pi^{\prime}$ in the plane with $U_{\mu}^{\pi}=\{1\}$ and $U_{\mu}^{\pi^{\prime}}=\{2\}$. Also, its sublink $L_{3}=L_{\mu}$ is the trivial link $T^{2}$ of Example 1, so $E_{1}\left(L_{\mu}\right)=0$ and $E_{2}\left(L_{\mu}\right)=\mathbf{Z} H_{\mu}$. Theorem (1.1) then states that

$$
\begin{aligned}
0 & =E_{1}\left(L_{\mu}\right)+\left(\left(\prod_{i<\mu} t_{i}^{l_{i}}\right)-1\right) E_{2}\left(L_{\mu}\right) \subseteq \phi E_{2}(L) \\
& \subseteq E_{1}\left(L_{\mu}\right)+I H_{\mu} \cdot E_{2}\left(L_{\mu}\right)=I H_{\mu},
\end{aligned}
$$

in which neither inclusion is an equality. Applying the lemma, it can be concluded that $\phi E_{2}(L) \subseteq\left(t_{1}-1\right)$, by considering the projection $\pi$, and $\phi E_{2}(L) \subseteq\left(t_{2}-1\right)$, by considering $\pi^{\prime}$, and hence $\phi E_{2}(L) \subseteq\left(t_{1}-1\right) \cap\left(t_{2}-1\right)$. Since $t_{1}-1$ and $t_{2}-1$ are relatively prime (see $\S 5)$, it follows that $\phi E_{2}(L) \subseteq\left(\left(t_{1}-1\right)\left(t_{2}-1\right)\right)$; this inclusion is in fact an equality. 
5. The properties (i'), (ii'), and (iii'). In this section these properties, mentioned in the introduction, are verified.

The property (i') is considered first. Since the ideals of a tame link form an ascending sequence, to verify ( $\left.i^{\prime}\right)$ it suffices to show that $\varepsilon E_{\mu}(L)=\mathbf{Z}$ whenever $L$ is a tame link with $\mu$ components; this is proven by induction on $\mu$. If $\mu=1$, this is a consequence of the property (i) stated in the introduction. Suppose that $\mu \geqslant 2$, and that (i') holds for all tame links of $\mu-1$ components. In particular, then $\bar{\varepsilon} E_{\mu-1}\left(L_{\mu}\right)$ $=\mathbf{Z}$, where $\bar{\varepsilon}: \mathbf{Z} H_{\mu} \rightarrow \mathbf{Z}$ is the augmentation map. Clearly $\varepsilon=\bar{\varepsilon} \phi: \mathbf{Z} \boldsymbol{H} \rightarrow \mathbf{Z}$, and hence by Theorem (1.1) $\mathbf{Z}=\bar{\varepsilon} E_{\mu-1}\left(L_{\mu}\right) \subseteq \bar{\varepsilon} \phi E_{\mu}(L)=\varepsilon E_{\mu}(L)$.

A similar inductive argument, using the second inclusion of Theorem (1.1) rather than the first, can be used to show that $\varepsilon E_{k}(L)=0$ (i.e., $E_{k}(L) \subseteq I H$ ) whenever $L$ is a tame link of $\mu>k$ components.

Proofs of the other two properties require more preparation.

In [2, p. 189] it is shown that if $\mu \geqslant 1$ and $H$ is a free abelian group with basis $t_{1}, \ldots, t_{\mu}$ then there is an exact sequence

$$
Y_{2} \stackrel{\alpha_{2}}{\rightarrow} Y_{1} \stackrel{\alpha_{1}}{\rightarrow} I H \rightarrow 0
$$

of $\mathbf{Z H}$-modules of the following form: $Y_{1}$ and $Y_{2}$ are free $\mathbf{Z H}$-modules with bases $\left\{b_{i} \mid 1 \leqslant i \leqslant \mu\right\}$ and $\left\{b_{i j} \mid 1 \leqslant i<j \leqslant \mu\right\}$, respectively, $\alpha_{1}\left(b_{i}\right)=t_{i}-1$ whenever $1 \leqslant$ $i \leqslant \mu$, and $\alpha_{2}\left(b_{i j}\right)=\left(t_{i}-1\right) b_{j}-\left(t_{j}-1\right) b_{i}$ whenever $1 \leqslant i<j \leqslant \mu$. (If $\mu=1$ then $Y_{2}=0$.)

From this, two properties of the elements $t_{1}-1, \ldots, t_{\mu}-1$ of $\mathbf{Z H}$ can be readily concluded, namely: if $i \neq j$ then $t_{i}-1$ and $t_{j}-1$ are relatively prime, and if $\mathbf{Z H}$ is considered as an algebra over $\mathbf{Z}$ then $\left\{t_{1}-1, \ldots, t_{\mu}-1\right\}$ is an algebraically independent subset (i.e., if $\mathbf{Z}\left[y_{1}, \ldots, y_{\mu}\right]$ is the ring of polynomials with integer coefficients in the indeterminates $y_{1}, \ldots, y_{\mu}$, then the (unique) homomorphism $\mathbf{Z}\left[y_{1}, \ldots, y_{\mu}\right] \rightarrow \mathbf{Z} H$ which takes each $y_{i}$ to $t_{i}-1$ is injective.)

A consequence of the latter property is

LeMMA (5.1). Let $H$ be a free abelian group with basis $t_{1}, \ldots, t_{\mu}$, and suppose that $x \in \mathbf{Z} H, n, p_{1}, \ldots, p_{\mu} \geqslant 0, p=\Sigma_{i} p_{i}>0, y=\Pi_{i}\left(t_{i}-1\right)^{p_{i}}$, and $x y \in(I H)^{n+p}$. Then $x \in(I H)^{n}$.

Proof. There is a $u_{0}$ it $u \in \mathbf{Z H}$ such that $u x$ is a polynomial (with integer coefficients) in $t_{1}, \ldots, t_{\mu}\left[1\right.$, p. 117]. Since $t_{i}=\left(t_{i}-1\right)+1 \forall i, u x$ is also a polynomial in $t_{1}-1, \ldots, t_{\mu}-1$, as is $u x y$.

Since $\left\{t_{1}-1, \ldots, t_{\mu}-1\right\}$ is algebraically independent, $u x$ and $u x y$ can be represented uniquely as sums of (nonzero) integer multiples of monomials ${ }^{3}$ in $t_{1}-1, \ldots, t_{\mu}-1$, without any monomial appearing more than once in either sum. By uniqueness, the representation of $u x y$ is obtained from that of $u x$ by multiplying by $y$. By hypothesis, each monomial appearing in the representation of $u x y$ is of total degree at least $n+p$, so each monomial appearing in the representation of $u x$ is of total degree at least $n$. Hence $u x \in(I H)^{n}$, so $x=u x u^{-1} \in(I H)^{n}$. Q.E.D.

\footnotetext{
${ }^{3} \mathrm{We}$ consider 1 , and no other integer, as a monomial.
} 
It is convenient to broaden the notation used heretofore. If $L=K_{1} \cup \cdots \cup K_{\mu}$ $\subseteq S^{3}$ and $1 \leqslant j \leqslant \mu$ then let $L_{j}$ be the sublink $L_{j}=K_{1} \cup \cdots \cup K_{j-1} \cup K_{j+1}$ $\cup \cdots \cup K_{\mu}=L-K_{j}$ of $L$, let $G_{j}=\pi_{1}\left(S^{3}-L_{j}\right)$ be the group of $L_{j}$ and $H_{j}$ its abelianization, let $t_{1}, \ldots, t_{j-1}, t_{j+1}, \ldots, t_{\mu} \in H_{j}$ be the preferred basis elements constructed in \$2, and let $\phi_{j}: \mathbf{Z} H \rightarrow \mathbf{Z} H_{j}$ be the (unique) ring homomorphism such that $\phi_{j}\left(t_{i}\right)=t_{i}$ whenever $i \neq j$ and $\phi_{j}\left(t_{j}\right)=1$. Then from Theorem (1.1) it can be concluded that

$$
E_{k-1}\left(L_{j}\right)+\left(\left(\prod_{i \neq j} t_{i}^{l\left(K_{i}, K_{j}\right)}\right)-1\right) E_{k}\left(L_{j}\right) \subseteq \phi_{j} E_{k}(L) \subseteq E_{k-1}\left(L_{j}\right)+I H_{j} \cdot E_{k}\left(L_{j}\right)
$$

whenever $k \geqslant 1$ and $1 \leqslant j \leqslant \mu$.

Property (iii') may now be verified, that is, that $E_{k}(L) \subseteq(I H)^{\mu-k}$ whenever $L$ is a tame link of $\mu$ components and $\mu>k \geqslant 1$. If $\mu=2$ then necessarily $k=1$, and $E_{1}(L) \subseteq I H$ by the property $\left(1_{2}\right)$ stated in the introduction. The proof proceeds by induction on $\mu$. By inductive hypothesis

$$
E_{k-1}\left(L_{j}\right) \subseteq\left(I H_{j}\right)^{\mu-1-(k-1)}=\left(I H_{j}\right)^{\mu-k}
$$

and

$$
E_{k}\left(L_{j}\right) \subseteq\left(I H_{j}\right)^{\mu-k-1}
$$

whenever $1 \leqslant j \leqslant \mu$. Hence by Theorem (1.1),

$$
\phi_{j} E_{k}(L) \subseteq E_{k-1}\left(L_{j}\right)+I H_{j} \cdot E_{k}\left(L_{j}\right) \subseteq\left(I H_{j}\right)^{\mu-k}=\phi_{j}\left((I H)^{\mu-k}\right)
$$

whenever $1 \leqslant j \leqslant \mu$. Suppose $x \in E_{k}(L)$; then $\phi_{1}(x) \in \phi_{1}\left((I H)^{\mu-k}\right)$, so

$$
x \in(I H)^{\mu-k}+\operatorname{ker} \phi_{1}=(I H)^{\mu-k}+\left(t_{1}-1\right)
$$

that is, $x=x_{1}+\left(t_{1}-1\right) y_{1}$ for some $x_{1} \in(I H)^{\mu-k}$ and $y_{1} \in \mathbf{Z} H$. If $k=\mu-1$ then this shows that $x \in I H=(I H)^{\mu-k}$. If $k<\mu-1$ then

$$
\phi_{2}(x)=\phi_{2}\left(x_{1}\right)+\phi_{2}\left(y_{1}\right)\left(t_{1}-1\right) \in\left(I H_{2}\right)^{\mu-k},
$$

and also

$$
\phi_{2}\left(x_{1}\right) \in \phi_{2}\left((I H)^{\mu-k}\right)=\left(I H_{2}\right)^{\mu-k}
$$

so

$$
\phi_{2}\left(y_{1}\right)\left(t_{1}-1\right) \in\left(I H_{2}\right)^{\mu-k} .
$$

Applying Lemma (5.1), it can be concluded that

$$
\phi_{2}\left(y_{1}\right) \in\left(I H_{2}\right)^{\mu-k-1}=\phi_{2}\left((I H)^{\mu-k-1}\right) \text {, }
$$

and hence

$$
y_{1} \in(I H)^{\mu-k-1}+\operatorname{ker} \phi_{2}=(I H)^{\mu-k-1}+\left(t_{2}-1\right),
$$

that is, $y_{1}=y_{1}^{\prime}+y_{1}^{\prime \prime}\left(t_{2}-1\right)$ for some $y_{1}^{\prime} \in(I H)^{\mu-k-1}$ and $y_{1}^{\prime \prime} \in \mathbf{Z} H$. Then

$$
x=x_{1}+y_{1}\left(t_{1}-1\right)=x_{2}+y_{2}\left(t_{1}-1\right)\left(t_{2}-1\right),
$$


where $x_{2}=x_{1}+y_{1}^{\prime}\left(t_{1}-1\right) \in(I H)^{\mu-k}$ and $y_{2}=y_{1}^{\prime \prime} \in \mathbf{Z} H$. If $k=\mu-2$, this shows that $x \in(I H)^{2}=(I H)^{\mu-k}$. If $k<\mu-2$, then the same argument (essentially) can be used, repeatedly, to show that whenever $2 \leqslant i \leqslant \mu-k$ there are $x_{i} \in(I H)^{\mu-k}$ and $y_{i} \in \mathbf{Z} H$ such that $x=x_{i}+y_{i} \cdot \Pi_{j=1}^{i}\left(t_{j}-1\right)$. In particular,

$$
x=x_{\mu-k}+y_{\mu-k} \cdot \prod_{j=1}^{\mu-k}\left(t_{j}-1\right) \in(I H)^{\mu-k} .
$$

Since $x \in E_{k}(L)$ was arbitrary, this shows that $E_{k}(L) \subseteq(I H)^{\mu-k}$.

Property (ii') differs from (i') and (iii') in that it is not, strictly speaking, a consequence of Theorem (1.1), though part of the proof of Theorem (1.1) is used in its verification.

LemMA (5.2). Let $H$ be a free abelian group with basis $t_{1}, \ldots, t_{\mu}, \mu \geqslant 2$, and let $J \subseteq \mathbf{Z} H$ be the ideal generated by the products $\left(t_{p}-1\right)\left(t_{q}-1\right), p \neq q$. Suppose $A_{1}, \ldots, A_{\mu}$ are ideals of $\mathbf{Z} H, x \in \mathbf{Z} H, 1 \leqslant i \leqslant \mu$, and $x\left(t_{i}-1\right) \in J+\sum_{j=1}^{\mu} A_{j}$. $\left(t_{j}-1\right)$. Then

$$
x \in\left(t_{1}-1, \ldots, t_{i-1}-1, t_{i+1}-1, \ldots, t_{\mu}-1\right)+A_{i} .
$$

Proof. Suppose that for $1 \leqslant j \leqslant \mu, A_{j}$ is the ideal of $\mathbf{Z} H$ generated by $\left\{a_{j k}\right\}$. It suffices to verify the lemma for $i=1$.

Suppose first that $\mu=2$. Then by hypothesis

$$
x\left(t_{1}-1\right)=x_{0}\left(t_{1}-1\right)\left(t_{2}-1\right)+\sum_{k} x_{1 k} a_{1 k}\left(t_{1}-1\right)+\sum_{k} x_{2 k} a_{2 k}\left(t_{2}-1\right)
$$

for some $x_{0}, x_{1 k}, x_{2 k} \in \mathbf{Z} H$. Since $t_{1}-1$ and $t_{2}-1$ are relatively prime and

$$
\left(\sum_{k} x_{2 k} a_{2 k}\right)\left(t_{2}-1\right)=\left(x-x_{0}\left(t_{2}-1\right)-\sum_{k} x_{1 k} a_{1 k}\right)\left(t_{1}-1\right)
$$

is divisible by $t_{1}-1, \Sigma_{k} x_{2 k} a_{2 k}=x_{1}\left(t_{1}-1\right)$ for some $x_{1} \in \mathbf{Z} H$. Thus by cancellation

$$
x=x_{0}\left(t_{2}-1\right)+x_{1}\left(t_{2}-1\right)+\sum_{k} x_{1 k} a_{1 k} \in\left(t_{2}-1\right)+A_{1} .
$$

Suppose now that $\mu>2$, and the lemma holds for free abelian groups of ranks $<\mu$. Let $H_{\mu}$ be the free abelian group with basis $t_{1}, \ldots, t_{\mu-1}$, and let $\phi_{\mu}$ : $H \rightarrow H_{\mu}$ be the group homomorphism given by $\phi_{\mu}\left(t_{j}\right)=t_{j}$ for $j<\mu$, and $\phi_{\mu}\left(t_{\mu}\right)=1$. Since

$$
\phi_{\mu}(x)\left(t_{1}-1\right) \in \phi_{\mu}(J)+\sum_{j=1}^{\mu-1} \phi_{\mu}\left(A_{j}\right) \cdot\left(t_{j}-1\right),
$$

by inductive hypothesis

$$
\phi_{\mu}(x) \in\left(t_{2}-1, \ldots, t_{\mu-1}-1\right)+\phi_{\mu}\left(A_{1}\right) \subseteq \mathbf{Z} H_{\mu} .
$$

Hence $x \in \phi_{\mu}^{-1}\left(\left(t_{2}-1, \ldots, t_{\mu-1}-1\right)+\phi_{\mu}\left(A_{1}\right)\right)=\left(t_{2}-1, \ldots, t_{\mu-1}-1\right)+A_{1}$ $+\operatorname{ker} \phi_{\mu}=\left(t_{2}-1, \ldots, t_{\mu}-1\right)+A_{1}$. Q.E.D.

As noted in $\$ 2$, any one of the relators in the presentation $\left\langle x_{i j} ; r_{i j}\right\rangle$ of $G=$ $\pi_{1}\left(S^{3}-L\right)$ is a consequence of the others. It is shown in [1, p. 105] that this 
implies that each row of the Alexander matrix $M$ of this presentation is a linear combination of the other rows. As a consequence of this, we obtain

Lemma (5.3). Suppose $2 \leqslant k \leqslant m$. Given a choice of $k$ columns and $k-1$ rows of $M$, let $X_{1}, \ldots, X_{m-k+1}$ be those $k \times k$ submatrices of $M$ which involve these chosen columns and rows. Then any one of $\operatorname{det} X_{1}, \ldots, \operatorname{det} X_{m-k+1}$ is in the ideal of $\mathbf{Z H}$ generated by the others.

Proof. Let $Y$ be the $(k-1) \times k$ submatrix of $M$ involving the chosen rows and columns. For $1 \leqslant i \leqslant m$ let $Y_{i}$ be the $k \times k$ matrix whose last $k-1$ rows are the rows of $Y$, and whose first row consists of those entries of the $i$ th row of $M$ that lie in the chosen columns.

Suppose that $1 \leqslant p \leqslant m-k+1$, and that $X_{p}$ involves the $q$ th row of $M$ in addition to the chosen rows. Since the $q$ th row of $M$ is a linear combination of the other rows of $M$, the multilinearity of the determinant (as a function of the rows of a matrix) implies that det $Y_{q}$ is a linear combination of the determinants of the $Y_{r}$, $r \neq q$. If the $r$ th row of $M$ is among the chosen rows, then $Y_{r}$ has two identical rows and, hence, det $Y_{r}=0$; thus det $Y_{q}$ is a linear combination of the determinants of those $Y_{r}, r \neq q$, such that the $r$ th row of $M$ is not among the chosen rows. Each such $Y_{r}$ is, up to permutation of its rows, one of $X_{1}, \ldots, X_{m-k+1}$, but not $X_{p}$; also $Y_{q}$ is, up to permutation of its rows, $X_{p}$. Thus det $X_{p}$ is a linear combination of the determinants of the $X_{s}, s \neq p$. Q.E.D.

A proof of (ii') may now be presented.

If $\mu=2$, then by property $\left(1_{2}\right)$ of the introduction,

$$
E_{1}(L)=\Delta_{1}(L) I H=\Delta_{1}(L) \cdot\left(t_{1}-1, t_{2}-1\right) .
$$

By $\left(2_{2}\right)$,

$$
\phi_{2}\left(\left(t_{1}-1\right) \Delta_{1}(L)\right)=\left(t_{1}^{l\left(K_{1}, K_{2}\right)}-1\right) \Delta_{1}\left(L_{\mu}\right)
$$

so

$$
\left(t_{1}-1\right) \Delta_{1}(L)-\left(t_{1}^{l\left(K_{1}, K_{2}\right)}-1\right) \Delta_{1}\left(L_{\mu}\right) \in \operatorname{ker} \phi_{2}=\left(t_{2}-1\right),
$$

that is, $\left(t_{1}-1\right) \Delta_{1}(L)-\left(t_{1}^{l\left(K_{1}, K_{2}\right)}-1\right) \Delta_{1}\left(L_{\mu}\right)$ is divisible by $t_{2}-1$. Since it is also divisible by $t_{1}-1$, and $t_{1}-1$ and $t_{2}-1$ are relatively prime, it must be divisible by the product $\left(t_{1}-1\right)\left(t_{2}-1\right)$. Thus

$$
\left(t_{1}-1\right) \Delta_{1}(L)-\left(t_{1}^{l\left(K_{1}, K_{2}\right)}-1\right) \Delta_{1}\left(L_{\mu}\right) \in J,
$$

so $\left(t_{1}-1\right) \Delta_{1}(L) \in C+J$. Similarly, $\left(t_{2}-1\right) \Delta_{1}(L) \in C+J$, and hence

$$
E_{1}(L)=\Delta_{1}(L) \cdot\left(t_{1}-1, t_{2}-1\right) \subseteq C+J .
$$

Proceeding inductively, suppose that $\mu>2$. The inductive hypothesis implies that $E_{\mu-2}\left(L_{i}\right) \subseteq \phi_{i}(J+C) \forall i \in\{1, \ldots, \mu\}$; clearly then

$$
E_{\mu-2}\left(L_{i}\right)+\left(\left(\prod_{j \neq i} t_{j}^{l\left(K_{i}, K_{j}\right)}\right)-1\right) E_{\mu-1}\left(L_{i}\right) \subseteq \phi_{i}(J+C) \quad \forall i \in\{1, \ldots, \mu\} .
$$

To verify that (ii') holds for $L$, it is necessary to show that $\operatorname{det} X \in J+C$ whenever $X$ is an $(n-\mu+1) \times(n-\mu+1)$ submatrix of $M$. The proof of this is split into two cases. 
Case 1. There is an $i_{1} \in\{1, \ldots, \mu\}$ and $j_{1}, j_{1}^{\prime} \in\left\{1, \ldots, j_{i_{1}}\right\}$ such that $X$ involves neither the column of $M$ corresponding to $e_{i_{1} j_{1}^{\prime}}$ nor the row of $M$ corresponding to the $i_{1} j_{1}$ th crossing in the projection. A simple counting argument indicates that either there is an $i_{2} \in\{1, \ldots, \mu\}$ such that $X$ does not involve the column of $M$ corresponding to $e_{i_{2} j}$ for some $j \in\left\{1, \ldots, j_{i_{2}}\right\}$, or else there is an $i_{2} \in\{1, \ldots, \mu\}$ such that $X$ involves both the column corresponding to $e_{i_{2} j}$ and the row corresponding to the $i_{2} j$ th crossing in the projection for every $j \in\left\{1, \ldots, j_{i_{2}}\right\}$. By Cases 1 and 2 of the final argument of the proof of Theorem (1.1),

$$
\phi_{i_{2}}(\operatorname{det} X) \in E_{\mu-2}\left(L_{i_{2}}\right)+\left(\left(\prod_{i \neq i_{2}} t_{i}^{l\left(K_{i}, K_{i_{2}}\right)}\right)-1\right) E_{\mu-1}\left(L_{i_{2}}\right)
$$

no matter which of these two properties is satisfied by $i_{2}$. Also, by Case 1 of the proof of Theorem (1.1), $\phi_{i_{1}}(\operatorname{det} X) \in E_{\mu-2}\left(L_{i_{1}}\right)$.

As noted above, we can conclude that $\phi_{i_{1}}(\operatorname{det} X) \in \phi_{i_{1}}(J+C)$ and $\phi_{i_{2}}(\operatorname{det} X) \in$ $\phi_{i_{2}}(J+C)$. From the former it follows that

$$
\operatorname{det} X \in J+C+\operatorname{ker} \phi_{i_{1}}=J+C+\left(t_{i_{1}}-1\right) \text {, }
$$

that is, there are $y \in J+C$ and $z \in \mathbf{Z} H$ with $\operatorname{det} X=y+\left(t_{i_{1}}-1\right) z$. Then $\phi_{i_{2}}(\operatorname{det} X)=\phi_{i_{2}}(y)+\left(t_{i_{1}}-1\right) \phi_{i_{2}}(z)$. Since $\phi_{i_{2}}(\operatorname{det} X)$ and $\phi_{i_{2}}(y)$ are both elements of $\phi_{i_{2}}(J+C)=\phi_{i_{2}}(J)+\phi_{i_{2}}(C)$, so is $\left(t_{i_{1}}-1\right) \phi_{i_{2}}(z)$.

Let $C_{i_{1}} \subseteq \mathbf{Z H}$ be the ideal generated by the set of elements of the form

$$
\frac{t_{i_{1}}^{l\left(K_{i_{1}}, K_{i}\right)}-1}{t_{i_{1}}-1}
$$

for $i \neq i_{1}$. (This expression denotes that element of $\mathbf{Z} H$ whose product with $t_{i_{1}}-1$ is $t_{i_{1}}^{l\left(K_{i_{1}}, K_{i}\right)}-1$.) Then by Lemma (5.2)

$$
\phi_{i_{2}}(z) \in \phi_{i_{2}}\left(\left(t_{1}-1, \ldots, t_{i_{1}-1}-1, t_{i_{1}+1}-1, \ldots, t_{\mu}-1\right)+C_{i_{1}}\right),
$$

and hence

$$
\begin{aligned}
z \in\left(t_{1}-1, \ldots, t_{i_{1}-1}\right. & \left.-1, t_{i_{1}+1}-1, \ldots, t_{\mu}-1\right)+C_{i_{1}}+\operatorname{ker} \phi_{i_{2}} \\
& =\left(t_{1}-1, \ldots, t_{i_{1}-1}-1, t_{i_{1}+1}-1, \ldots, t_{\mu}-1\right)+C_{i_{1}} .
\end{aligned}
$$

Thus $\left(t_{i_{1}}-1\right) z \in J+C$ and, hence, $\operatorname{det} X=y+\left(t_{i_{1}}-1\right) z \in J+C$.

Case 2. For any $i \in\{1, \ldots, \mu\}$, either $X$ involves the column of $M$ corresponding to $e_{i j}$ for every $j \in\left\{1, \ldots, j_{i}\right\}$, or $X$ involves the row of $M$ corresponding to the $i j$ th crossing in the projection for every $j \in\left\{1, \ldots, j_{i}\right\}$. Choose $i_{1} \in$ $\{1, \ldots, \mu\}$ and $j \in\left\{1, \ldots, j_{i_{1}}\right\}$ such that $X$ does not involve the column of $M$ corresponding to $e_{i_{1} j}$, and let $X=X_{1}, X_{2}, \ldots, X_{m-n+\mu}$ be the $(n-\mu+1) \times(n-$ $\mu+1)$ submatrices of $M$ which involve the same columns as $X$ and involve every row of $M$ involved by $X$, except possibly the one corresponding to the $i_{1} j$ th crossing in the projection. Then each $X_{i}, i \geqslant 2$, falls under Case 1 and, hence, $\operatorname{det} X_{i} \in J+C$ whenever $i \geqslant 2$. By Lemma (5.3), det $X$ is an element of the ideal of $\mathbf{Z} H$ generated by the determinants of the $X_{i}, i \geqslant 2$; hence $\operatorname{det} X \in J+C$. Q.E.D. 
Before closing out this section, a final comment may be in order. The obvious analogy between the properties (i), (ii) and (iii) and the properties (i'), (ii'), and (iii') may give the impression that "everything that can be said about the first elementary ideal can be said about the higher ideals". This is not true. To illustrate this point, consider a link $L=K_{1} \cup K_{2}$ such that $l\left(K_{1}, K_{2}\right)=0$. From Torres' relation $\left(2_{2}\right)$ it can be concluded that $\Delta_{1}(L)\left(t_{1}, 1\right)=\phi_{2} \Delta_{1}(L)=0$, and also that $\Delta_{1}(L)\left(1, t_{2}\right)=\phi_{1} \Delta_{1}(L)=0$, so that $\Delta_{1}(L)$ is divisible by the product $\left(t_{1}-1\right)\left(t_{2}-1\right)$; in particular, $\Delta_{1}(L) \in(I H)^{2}$, and hence $E_{1}(L)=\Delta_{1}(L) \cdot I H \subseteq$ $(I H)^{3}$. (This can also be concluded from the property (ii).) However, it is not true, in general, that $E_{\mu-1}(L) \subseteq(I H)^{3}$ if $L$ is a link of $\mu \geqslant 3$ components and all the linking numbers of the various components of $L$ with each other are zero, as can be seen by considering Example 5 of $\$ 3$.

6. The link polynomials. A simple consequence of the first inclusion of Theorem $(1.1)$ is

COROllary (6.1). In the situation of the statement of Theorem (1.1), $\Delta_{k}(L)\left(t_{1}, \ldots, t_{\mu-1}, 1\right)=\phi \Delta_{k}(L)$ divides both $\Delta_{k-1}\left(L_{\mu}\right)$ and $\left(\left(\Pi_{i<\mu} t_{i}^{l}\right)-1\right) \Delta_{k}\left(L_{\mu}\right)$ for any $k \geqslant 1$.

(This follows immediately from the definition of the g.c.d. of an ideal of $\mathbf{Z H}_{\mu}$.)

The second inclusion of Theorem (1.1) cannot be used in a similar manner, since $\phi \Delta_{k}(L)$ need not be a g.c.d. of $\phi E_{k}(L)$ in $\mathbf{Z} H_{\mu}$.

An inductive proof can be constructed very easily, using Corollary (6.1) and the property (i) of the introduction, of a property which may reasonably be called property (i"): namely, that $\varepsilon \Delta_{k}(L)=\Delta_{k}(L)(1, \ldots, 1)= \pm 1$ whenever $L$ is a tame link of $\mu \leqslant k$ components.

Furthermore, Corollary (6.1) can be generalized to state: if $1 \leqslant i \leqslant \mu$ then $\Delta_{k}(L)\left(t_{1}, \ldots, t_{i-1}, 1, t_{i+1}, \ldots, t_{\mu}\right)$ divides both $\Delta_{k-1}\left(L_{i}\right)$ and

$$
\left(\left(\prod_{i \neq j} t_{j}^{l\left(K_{i}, K_{j}\right)}\right)-1\right) \Delta_{k}\left(L_{i}\right),
$$

for any $k \geqslant 1$, just as Theorem (1.1) was generalized in §5. A simple inductive argument, using this statement and the fact that for tame links of two components $\pm \varepsilon \Delta_{1}(L)$ is the linking number, may be used to show: $\Delta_{\mu-1}(L)(1, \ldots, 1)=$ $\varepsilon \Delta_{\mu-1}(L)$ divides the linking number of any two components of $L$ if $L$ is a tame link of $\mu$ components. In the string of analogies, this is property (ii").

Unfortunately, this property (ii") is highly inaccurate. For instance, consider that the link of Example 5 of $\S 3$ has $\Delta_{2}=\Delta_{\mu-1}=1$, even though the linking numbers of its components with each other are all zero.

Even worse is the failure of any "property (iii")". For the links of Example 6 of $\$ 3$ show that for any $\mu \geqslant 3$ there is a link of $\mu$ components with $\Delta_{2}=1$.

In sum, Theorem (1.1) and the properties (i'), (ii'), and (iii') are reflected only partially by the link polynomials. This is symptomatic of the loss of information involved in the passage from the ideals to their g.c.d.s. 


\section{BIBLIOGRAPHY}

1. R. H. Crowell and R. H. Fox, Introduction to knot theory, Springer-Verlag, Berlin and New York, 1977.

2. S. Mac Lane, Homology, Springer-Verlag, Berlin and New York, 1967.

3. D. Rolfsen, Knots and links, Publish or Perish, Berkeley, Calif., 1976.

4. H. Seifert, Uber das Geschlecht von Knoten, Math. Ann. 110 (1934), 571-592.

5. G. Torres, On the Alexander polynomial, Ann. of Math. (2) 57 (1953), 57-89.

6. L. Traldi, On the determinantal ideals of link modules and a generalization of Torres' second relation, Ph.D. Dissertation, Yale Univ., New Haven, Conn., 1980.

Department of Mathematics, Lafayette College, Easton, Pennsylvania 18042 